\title{
Do Anthropometric Measures Influence Torso Muscle Endurance Profiles of Children Aged 7 to 14?
}

\author{
Aleksandar Dejanovic $^{1}$, Erin Harvey ${ }^{2}$, Jordan Andersen $^{3}$, Stuart McGill $^{3}$ \\ ${ }^{1}$ Department of Biochemical and Medicine Science, University of Novi Pazar, Novi Sad, Serbia \\ ${ }^{2}$ Department of Statistics and Actuarial Science, Faculty of Mathematics, University of Waterloo, \\ Waterloo, Canada \\ ${ }^{3}$ Department of Kinesiology, Faculty of Applied Health Sciences, University of Waterloo, \\ Waterloo, Canada \\ Email: mcgill@uwaterloo.ca
}

Received August $1^{\text {st }}, 2012$; revised September $4^{\text {th }}, 2012$; accepted September $16^{\text {th }}, 2012$

\begin{abstract}
The aim of this study was to investigate whether torso endurance scores were linked with anthropometric variables in children and to develop a normative scale of anthropometric measures in children in both genders for clinical assessment, rehabilitation, physical education targets and young athletic training purposes. It was hypothesized that changes in anthropometric measures through ages 7 to 14 influence endurance scores in both subsets. It was also hypothesized that boys and girls differ in the relationships between torso muscle endurance and anthropometric measures. Reduced torso muscle endurance has been identified as a potential personal risk factor for developing low back pain and decreased athletic performance. However, torso muscle endurance data in children is lacking. Further, given that endurance tests require postures where the body is supported horizontally, it makes sense that anthropometric variables would influence endurance. Isometric torso muscle endurance scores established through four tests were performed in random order by healthy children. These were correlated with anthropometric dimensions. Seven hundred and fifty-three children from one elementary school (394 boys and 359 girls) were grouped into 8 age strata (7 to 14). Each age stratum had different number of participants for boys and girls. Four tests established isometric torso muscle endurance: Biering-Sorensen test for extensor endurance, flexor endurance test and right and left side bridge tests. The mean, standard deviation of the endurance tests and anthropometric measures were determined for each gender/age strata. The Pearson product-moment correlation coefficients were determined between the anthropometric dimensions and isometric torso endurance scores for each gender/age strata. Variance in endurance scores were not well explained by anthropometric measures. Variables other than segment length and circumference influence torso endurance as children grow and develop. Given links to future back pain and athletic performance, more investigation would be justified.
\end{abstract}

Keywords: Anthropometric Measures; Torso Muscle Endurance Tests; Correlation; Children

\section{Introduction}

Isometric muscle endurance of the torso has been reported in children (Dejanovic, 2006), adolescents (Salminen et al., 1992) and adults (Biering-Sorensen, 1984; McGill et al., 1999). These values are important as they have been identified as potential risk factors for developing low back pain and decreased athletic performance. Given that endurance test postures require the body to be supported horizontally, it makes sense that anthropometrics would influence endurance.

It has been shown that correlations between some anthropometric measures and fitness variables, such as strength, are important. Evidence indicates that body weight and height, among children, are correlated with grip strength across several populations (Everett \& Sills, 1952; Johnson, 1925; Metheny, 1941). Further, there is general agreement that strength and endurance are also important for motor development in children. For example, Rarick and Dobbins (1975) emphasized strength and its relationship to body size as major factors in physical performance and that excess of body fat decreased motor performances in children aged 6 - 9. Gabbard and Patterson (1980), however, noted low correlations between muscular endurance, body weight and height among children aged 3 - 5. Specifically, a several reports suggested that endurance scores were lower in tall and high body mass children (Docherty \& Gaul, 1991; Slaughter et al., 1977). Furthermore, Jürimäe and Jürimäe (2000) found no correlation between Eurofit test results and body height in boys while there was a relationship in girls. In fact, longitudinal body dimensions in pre-pubertal girls had more influences on motor ability tests when compared with boys.

The current study was conducted to investigate the links between selected anthropometric measures and isometric torso muscle endurance in boys and girls aged 7 to 14 . Another goal of this study was to develop a database of these measures for children. It was hypothesized that changes in torso endurance scores are associated with anthropometric measures. It was also hypothesized that boys and girls differ in the relationship between torso muscle endurance and anthropometric measures.

\section{Methods}

Anthropometric measurements and torso muscle endurance were obtained from boys and girls at an elementary school us- 
ing previously established protocols (Dejanovic et al., 2012) but which are repeated here. First, confirm that you have the correct template for your paper size. This template has been tailored for output on the custom paper size $(21 \mathrm{~cm} * 28.5 \mathrm{~cm})$.

\section{Subjects}

Seven hundred and fifty-three children (394 boys and 359 girls) aged 7 - 14 years from a Serbian elementary school took part. The testing and data collection methods were presented to, and approved by, the Parents' Committee of the elementary school, City of Novi Sad, Republic of Serbia, as well as from the Teaching and Scientific Council, Faculty of Sport and Physical Education in Nis, Department for Applied Kinesiology, University of Nis, Republic of Serbia. All parents signed informed consent documents prior to data collection. To reduce risk of injury and psychological distress, each test was explained and demonstrated in front of the children. The inclusion criteria for participants were: aged from 7 to 14 years; no current or previous history of neurological or orthopaedic problems of the spine and hips; no upper or lower musculoskeletal disorders or injuries; prior to testing, all subjects needed to feel healthy, which was confirmed verbally before the testing session.

\section{Instrumentation}

For the anthropometric measurements, the following standard instruments were used: Segment lengths were measured using the Martin anthropometer, scaled $0-200 \mathrm{~cm}$ with a reading accuracy of $0.1 \mathrm{~cm}$. A balance beam scale, accurate to 0.1 $\mathrm{kg}$, measured participants' weight. Measuring tape $150 \mathrm{~cm}$ in length was used to measure segment circumferences, accurate to $0.1 \mathrm{~cm}$. Finally, Martin sliding calipers with ranges of $0-40$ $\mathrm{cm}$ and $0-20 \mathrm{~cm}$ were used for segment girth measurements, also accurate to $0.1 \mathrm{~cm}$.

\section{Data Collection}

\section{Torso Muscle Endurance Tests}

Four tests were used to measure isometric torso muscle endurance after McGill et al. (1999): Biering-Sorensen test, torso flexor endurance test and right and left lateral torso test. McGill et al. found these tests to be reliable with a reliability coefficient $>0.97$ when tested consecutively over a five-day period. Evans and colleagues (2007) documented high reliability of the lateral endurance tests.

Back Extension Test (BET): The Biering-Sorensen test was used to measure back torso muscle endurance. Participants lay on their front with their hips (anterior superior iliac spine (ASIS) $)$ in-line with the edge of a test bench $(150 \times 110 \times 50$ $\mathrm{cm}$ ) so that the upper body was cantilevered out over the end, holding their arms across the chest. To keep the participant in place, the pelvis, knees and hips were secured with padded straps or a research assistant held both ankles. The test ended when the participant could no longer maintain a horizontal position or when they reached 300 seconds. During the test, participants were allowed to be verbally corrected twice to maintain a horizontal position and the test ended on the third correction.

Flexor Endurance Test (FET): Participants adopted a sit-up position with arms placed across the chest. The feet were se- cured under toe straps or by a researcher and the back began resting against a jig angled $50^{\circ}$ from the floor with knees and hips flexed at $90^{\circ}$. To begin, the jig was pulled back $10 \mathrm{~cm}$ while the subject held this position for as long as possible. The test was stopped when the participants' back touched the jig or when a maximum time of 300 seconds was reached.

Left and Right Side Bridge Tests (LS \& RS): Lateral torso muscle endurance was tested with the subject lying in the full side-bridge position. Legs were extended, the top foot was placed in the front of the lower foot for support and the participant was asked to bridge up with the elbow while holding their hips off the floor. The test was terminated when the subject lost a straight back posture or when a maximum time of 300 seconds was reached.

\section{Anthropometric Measures}

The following parameters were used to evaluate the anthropometric measurements: 1) longitudinal dimensions-body height, sitting height, upper arm length, arm length and leg length; 2) transversal dimensions - shoulder diameter (biacromial diameter), pelvic width (bicristal diameter) and knee diameter; 3) circumferential dimensions - upper arm, forearm, chest, waist, hips, upper leg and calf; and 4) body weight. Body weight was measured to the closest $0.5 \mathrm{~kg}$. All other measurements were taken to the closest $0.1 \mathrm{~cm}$. Anthropometric measurements were carried out in accordance with the guidelines of the International Biological Program (Lohman et al., 1988).

Body height was measured with participants standing barefoot in an upright position. A measurement was taken from the floor to the top of the participant's head in a neutral position. Sitting height was done with participants in a flat-seated chair with his or her back in a neutral position. A measurement was taken from the seat of the chair to the top of the participants' head. For the upper arm, a measurement was taken from the acromion to the olecranon with the elbow flexed at $90^{\circ}$ and the palm facing up. Arm length was taken from the acromion to the tip of the middle finger when the arm was in front of the participant parallel to the ground with the palm facing up. Leg length was taken from the floor to the ASIS when the participant was standing upright.

Shoulder and pelvic diameter were measured with the Martin sliding caliper. With the participant in an upright standing position, the anthropometer was placed on the outer part of both acromia for the shoulders and at the top of the iliac crest for the pelvis. Knee diameter was taken around the level of the epicondyles of the femur with the participant in a seated position and the knee bent to $90^{\circ}$.

All circumference measurements were carried out with the participants in an upright standing posture, except for the calf that was done in a seated position. The upper arm measurement was taken midway between the acromion and the olecranon. The forearm, upper leg and calf measurements were taken at the level of greatest circumference of the segment. Circumference of the chest was taken from between the attachment of the 3 rd and 4 th ribs around to the sternum. A measurement was taken at the end of a normal exhalation. Waist circumference was taken at the level of the umbilicus and hip circumference was measured at the greatest circumference of the gluteal muscles.

Body weight was measured with the participant in a quiet, upright standing posture. 


\section{Statistical Analysis}

Torso endurance test scores were not normally distributed for boys or girls, determined by the Kolmogorov-Smirnov test in SPSS Statistics 20.0. Stem-and-leaf plots suggested that this was likely due to a ceiling effect of endurance scores (i.e. numerous children reached the $300 \mathrm{~s}$ time limit for each test). Spearman rank-order correlation was used to determine the strength of relationships between torso endurance scores and anthropometric measurements for each gender. The participants were then separated into 8 age groups (ages 7 through 14). For boys, the numbers of participants in each age group were: $7(\mathrm{n}=30)$, $8(\mathrm{n}=35), 9(\mathrm{n}=58), 10(\mathrm{n}=42), 11(\mathrm{n}=59), 12(\mathrm{n}=49), 13$ $(n=63)$ and $14(n=58)$. For girls, the numbers of participants were: $7(n=41), 8(n=38), 9(n=50), 10(n=42), 11(n=58)$, $12(\mathrm{n}=43), 13(\mathrm{n}=45)$ and $14(\mathrm{n}=42)$. This division was done to determine whether there were any differences in torso endurance scores and anthropometric measures at different ages between genders. Spearman correlations were determined between endurance times and anthropometrics for each age group of both genders.

Means and standard deviations were calculated for each individual age group by gender for all of the torso endurance and anthropometric measures. The Kruskal-Wallis test was used to determine whether there was a significant effect of gender on the 4 torso endurance scores $(\alpha=0.0125$ with Bonferroni correction). The Mann-Whitney $U$ statistic was then used to determine the loci of these differences, with the alpha value Bonferroni corrected to 0.007 .

\section{Results}

Means and standard deviations of torso endurance scores and anthropometric measures are presented in Tables 1(a)-(d) for the 8 separate age groups of boys and girls.

\section{Boys}

Examining the relationships between endurance variables and anthropometric variables, independent of age, Spearman correlations were strongest between BET and longitudinal anthropometric measures; however, while statistically significant, the highest $\mathrm{R}$ value was 0.28 , meaning that very little variance was explained. Figure 1 illustrates this notion with an example plotting all endurance scores with height. Among each torso endurance test, the highest correlations were also in the longitudinal dimension; however, relationships between FET, LS and RS and anthropometrics, though significant, were also very low (Table 2(a)).

When examining each age group as a cluster, Spearman correlations showed a different trend. Of all the anthropometric measurements, circumferential dimensions were most correlated with the torso endurance scores for boys. Knee diameter was correlated with the greatest number of endurance tests and across the highest number of age groups (the largest being $r=$ 0.53 ). The next most correlated anthropometric variables were forearm circumference, upper leg circumference and body weight. Most of the correlations, especially those that were significant, were negative; however, correlations between the BET test for 14 year old boys and these 4 body measurements were all positive (Table 3(a)).

Changes in all anthropometric variables followed a linear trend when plotted against age; however, torso endurance test scores did not as there were several inflection points. For example, BET showed an initial peak at 9 years then a second peak at 13 years (Figure 2). FET, on the other hand, increased almost twofold from ages 7 to 8 in boys before it leveled off at age 9. A slight drop in abdominal torso endurance occurred at age 12 before it increased in ages 13 and 14. A similar trend exists in both lateral torso endurance tests, except the dips in

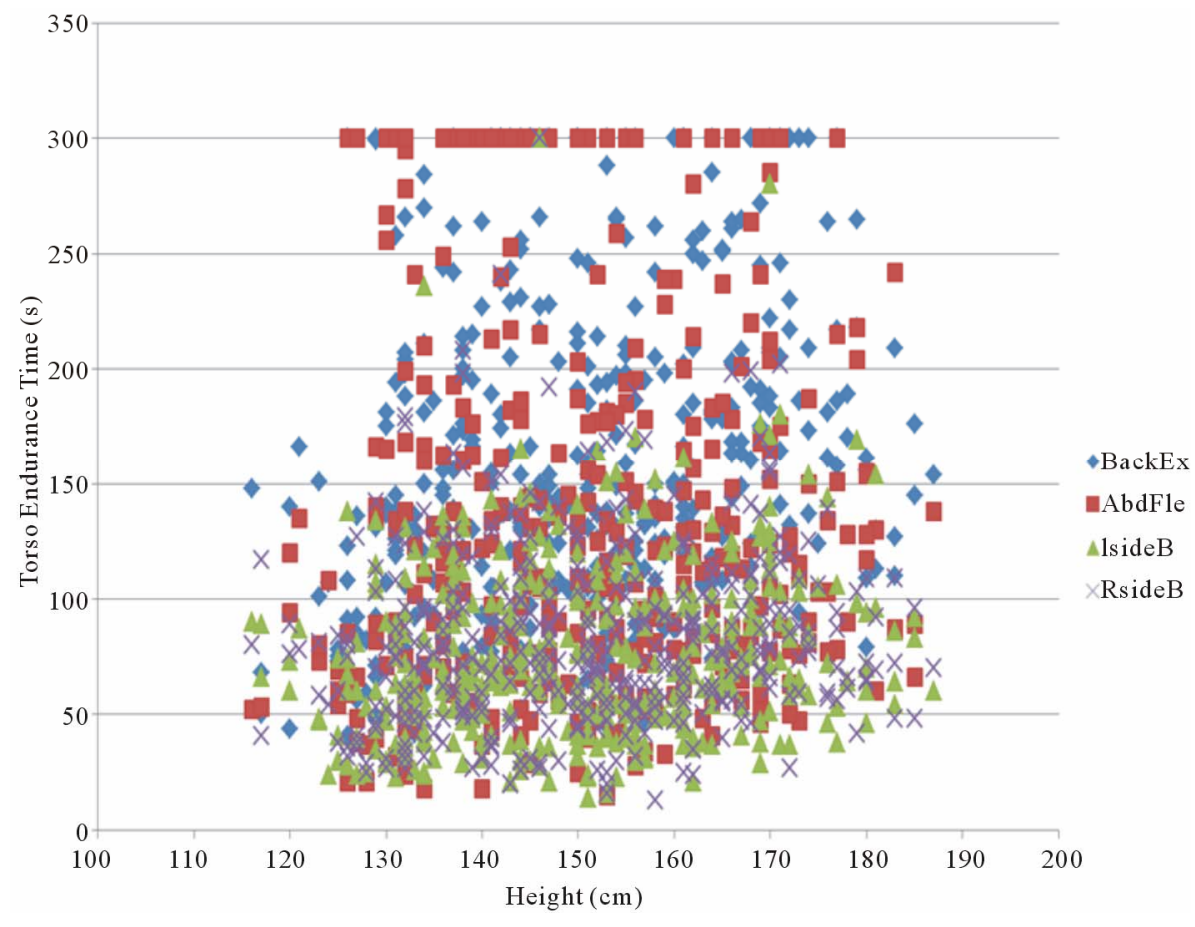

Figure 1.

A scatter plot of all endurance scores for boys and height illustrating no obvious links. 
Table 1.

(a) Mean and standard deviation of torso endurance tests for boys and girls of all age groups; (b) Mean and standard deviation of anthropometric longitudinal dimensions for boys and girls of all age groups; (c) Mean and standard deviation of anthropometric transversal dimensions and body weight for boys and girls of all age groups; (d) Mean and standard deviation of anthropometric circumferential dimensions for boys and girls of all age groups.

(a)

\begin{tabular}{|c|c|c|c|c|c|c|c|c|c|c|}
\hline \multirow{3}{*}{ Sex } & \multirow{3}{*}{ Age } & \multirow{3}{*}{$\mathrm{N}$} & \multicolumn{8}{|c|}{ Torso Endurance } \\
\hline & & & \multicolumn{2}{|c|}{ BET } & \multicolumn{2}{|c|}{ FET } & \multicolumn{2}{|c|}{ LS } & \multicolumn{2}{|c|}{ RS } \\
\hline & & & Mean & SD & Mean & $\mathrm{SD}$ & Mean & $\mathrm{SD}$ & Mean & SD \\
\hline \multirow{8}{*}{ Male } & 7 & 30 & 110.8 & 59.7 & 76.0 & 51.2 & 62.4 & 32.0 & 66.1 & 27.6 \\
\hline & 8 & 35 & 126.1 & 67.9 & 140.6 & 87.2 & 60.0 & 26.4 & 60.0 & 24.6 \\
\hline & 9 & 58 & 150.7 & 63.3 & 147.9 & 91.5 & 74.5 & 40.7 & 84.6 & 54.7 \\
\hline & 10 & 42 & 165.1 & 68.7 & 138.0 & 74.7 & 82.0 & 44.6 & 88.5 & 42.5 \\
\hline & 11 & 59 & 160.2 & 67.5 & 129.3 & 78.9 & 72.4 & 30.8 & 76.8 & 31.0 \\
\hline & 12 & 49 & 169.1 & 64.2 & 124.4 & 69.3 & 71.9 & 36.2 & 79.9 & 38.2 \\
\hline & 13 & 63 & 183.0 & 70.2 & 138.7 & 71.6 & 84.4 & 32.5 & 88.9 & 31.2 \\
\hline & 14 & 58 & 181.4 & 59.7 & 155.0 & 75.7 & 95.0 & 43.9 & 97.7 & 38.4 \\
\hline \multirow{8}{*}{ Female } & 7 & 41 & 111.0 & 44.5 & 96.6 & 75.9 & 56.9 & 21.5 & 59.1 & 22.1 \\
\hline & 8 & 38 & 137.0 & 64.6 & 100.7 & 81.0 & 44.7 & 24.7 & 53.9 & 24.6 \\
\hline & 9 & 50 & 191.7 & 62.9 & 168.6 & 95.3 & 84.5 & 38.9 & 77.6 & 43.0 \\
\hline & 10 & 42 & 202.1 & 65.6 & 149.1 & 81.4 & 85.9 & 38.9 & 96.0 & 46.8 \\
\hline & 11 & 58 & 182.0 & 67.8 & 111.0 & 69.3 & 77.5 & 39.1 & 76.1 & 27.9 \\
\hline & 12 & 43 & 210.4 & 56.9 & 126.2 & 64.2 & 68.5 & 32.1 & 64.9 & 24.9 \\
\hline & 13 & 45 & 206.3 & 57.9 & 148.2 & 79.2 & 75.7 & 29.3 & 80.3 & 34.0 \\
\hline & 14 & 42 & 197.6 & 72.7 & 140.8 & 72.3 & 86.0 & 32.2 & 86.0 & 35.3 \\
\hline \multicolumn{3}{|c|}{$\begin{array}{l}\text { Average difference between } \\
\text { genders across all age groups }\end{array}$} & ${ }^{\dagger}-23.9$ & ${ }^{\dagger} 14.9$ & ${ }^{\dagger} 1.1$ & ${ }^{\dagger} 21.3$ & $\dagger^{\dagger} .8$ & ${ }^{\dagger} 8.5$ & ${ }^{\dagger} 6.1$ & ${ }^{\dagger} 6.9$ \\
\hline
\end{tabular}

Note: ${ }^{\dagger}$ Positive values denote greater value from male population; Negative values show bias towards females; Torso endurance scores measured in seconds; BET_-back extension test (Biering-Sorensen); FET — flexor endurance test (sit-up posture); LS — left side bridge; RS — right side bridge.

\begin{tabular}{|c|c|c|c|c|c|c|c|c|c|c|c|c|}
\hline \multirow[b]{2}{*}{ Sex } & \multirow[b]{2}{*}{ Age } & \multirow[b]{2}{*}{$\mathrm{N}$} & \multicolumn{10}{|c|}{ Longitudinal Dimensions } \\
\hline & & & \multicolumn{2}{|c|}{ Body Height } & \multicolumn{2}{|c|}{ Sitting Height } & \multicolumn{2}{|c|}{ Leg Length } & \multicolumn{2}{|c|}{ Arm Length } & \multicolumn{2}{|c|}{ Upper Arm Length } \\
\hline \multirow{7}{*}{ Male } & 7 & 30 & 127.1 & 5.5 & 68.9 & 2.9 & 71.1 & 3.8 & 53.1 & 3.2 & 24.2 & 1.7 \\
\hline & 8 & 35 & 132.1 & 4.6 & 69.5 & 2.5 & 75.3 & 3.3 & 55.1 & 2.1 & 26.1 & 10.0 \\
\hline & 10 & 42 & 144.2 & 6.5 & 75.8 & 3.3 & 84.1 & 4.9 & 62.9 & 3.9 & 28.3 & 2.2 \\
\hline & 11 & 59 & 150.5 & 7.1 & 77.9 & 4.0 & 88.4 & 5.0 & 64.1 & 3.7 & 29.2 & 2.5 \\
\hline & 12 & 49 & 157.1 & 6.9 & 81.2 & 3.3 & 92.2 & 6.4 & 68.2 & 4.2 & 31.5 & 2.7 \\
\hline & 13 & 63 & 165.0 & 8.4 & 84.4 & 4.7 & 96.8 & 6.0 & 72.3 & 4.8 & 33.6 & 6.8 \\
\hline & 14 & 58 & 169.7 & 8.0 & 88.1 & 4.5 & 99.1 & 5.0 & 74.3 & 4.1 & 34.1 & 2.1 \\
\hline \multirow{7}{*}{ Female } & 7 & 41 & 126.8 & 5.1 & 68.9 & 2.7 & 70.9 & 3.9 & 52.7 & 3.2 & 23.8 & 1.6 \\
\hline & 9 & 50 & 138.0 & 6.2 & 72.2 & 3.1 & 78.6 & 4.4 & 56.8 & 3.2 & 25.6 & 1.9 \\
\hline & 10 & 42 & 144.7 & 8.4 & 76.2 & 4.3 & 84.5 & 5.8 & 62.9 & 4.2 & 29.1 & 2.1 \\
\hline & 11 & 58 & 151.2 & 7.9 & 79.0 & 4.2 & 87.8 & 5.3 & 65.3 & 4.1 & 30.3 & 2.1 \\
\hline & 12 & 43 & 158.5 & 7.0 & 82.5 & 3.8 & 91.2 & 5.4 & 69.3 & 3.8 & 32.0 & 2.1 \\
\hline & 13 & 45 & 162.6 & 6.0 & 84.5 & 3.9 & 94.6 & 4.6 & 70.6 & 3.8 & 32.6 & 1.9 \\
\hline & 14 & 42 & 165.0 & 7.0 & 86.9 & 3.7 & 94.5 & 4.8 & 71.8 & 3.6 & 33.4 & 1.7 \\
\hline \multicolumn{3}{|c|}{$\begin{array}{l}\text { Average difference between } \\
\text { genders across all age groups }\end{array}$} & ${ }^{\dagger} 0.8$ & ${ }^{\dagger} 2.0$ & ${ }^{\dagger}-0.1$ & ${ }^{\dagger} 0.8$ & ${ }^{\dagger} 1.2$ & ${ }^{\dagger} 1.6$ & ${ }^{\dagger} 0.4$ & ${ }^{\dagger} 1.3$ & ${ }^{\dagger} 0.04$ & ${ }^{\dagger} 0.8$ \\
\hline
\end{tabular}

Note: ${ }^{\dagger}$ Positive values denote greater value from male population; Negative values show bias towards females; Longitudinal dimensions measured in centimetres. 
(c)

\begin{tabular}{|c|c|c|c|c|c|c|c|c|c|c|}
\hline \multirow{3}{*}{ Sex } & \multirow{3}{*}{ Age } & \multirow{3}{*}{$\mathrm{N}$} & \multicolumn{6}{|c|}{ Transversal Dimensions } & \multicolumn{2}{|c|}{ Body Weight } \\
\hline & & & \multicolumn{2}{|c|}{ Shoulder Diameter } & \multicolumn{2}{|c|}{ Pelvis Diameter } & \multicolumn{2}{|c|}{ Knee Diameter } & \multirow{2}{*}{ Mean } & \multirow{2}{*}{ SD } \\
\hline & & & Mean & SD & Mean & SD & Mean & $\mathrm{SD}$ & & \\
\hline \multirow{8}{*}{ Male } & 7 & 30 & 27.6 & 1.5 & 19.2 & 1.1 & 8.3 & 0.7 & 26.4 & 4.5 \\
\hline & 8 & 35 & 28.7 & 1.5 & 19.7 & 1.1 & 8.5 & 0.7 & 30.1 & 4.7 \\
\hline & 9 & 58 & 30.0 & 1.5 & 20.6 & 1.3 & 8.8 & 0.7 & 35.1 & 6.4 \\
\hline & 10 & 42 & 30.5 & 3.8 & 21.6 & 1.7 & 8.7 & 0.9 & 39.0 & 9.1 \\
\hline & 11 & 59 & 32.2 & 2.1 & 21.7 & 1.8 & 9.8 & 1.0 & 44.7 & 10.9 \\
\hline & 12 & 49 & 34.0 & 2.0 & 23.4 & 1.7 & 9.6 & 0.9 & 48.4 & 10.3 \\
\hline & 13 & 63 & 35.3 & 2.3 & 23.6 & 1.7 & 9.9 & 0.9 & 54.4 & 12.1 \\
\hline & 14 & 58 & 35.6 & 2.5 & 24.7 & 2.5 & 10.0 & 0.8 & 57.0 & 9.1 \\
\hline \multirow{8}{*}{ Female } & 7 & 41 & 26.9 & 1.3 & 18.9 & 1.1 & 8.0 & 0.7 & 26.2 & 4.1 \\
\hline & 8 & 38 & 28.7 & 1.5 & 19.8 & 1.3 & 8.2 & 0.7 & 29.3 & 5.3 \\
\hline & 9 & 50 & 29.3 & 1.6 & 20.6 & 1.5 & 8.6 & 0.8 & 33.0 & 7.5 \\
\hline & 10 & 42 & 29.7 & 2.3 & 21.4 & 1.8 & 8.3 & 0.9 & 37.2 & 8.3 \\
\hline & 11 & 58 & 31.8 & 2.0 & 22.6 & 2.0 & 9.2 & 0.8 & 42.9 & 9.2 \\
\hline & 12 & 43 & 33.0 & 2.6 & 24.2 & 3.7 & 9.0 & 0.9 & 47.0 & 8.6 \\
\hline & 13 & 45 & 34.6 & 1.6 & 24.6 & 1.6 & 9.5 & 1.0 & 53.1 & 10.1 \\
\hline & 14 & 42 & 34.2 & 2.2 & 24.9 & 1.7 & 9.2 & 0.7 & 54.6 & 8.8 \\
\hline \multicolumn{3}{|c|}{$\begin{array}{l}\text { Average difference between genders } \\
\text { across all age groups }\end{array}$} & ${ }^{\dagger} 0.7$ & ${ }^{\dagger} 0.4$ & ${ }^{\dagger}-0.3$ & ${ }^{\dagger} 0.5$ & ${ }^{\dagger} 0.5$ & ${ }^{\dagger} 0.2$ & ${ }^{\dagger} 1.5$ & ${ }^{\dagger} 0.7$ \\
\hline
\end{tabular}

Note: ${ }^{\dagger}$ Positive values denote greater value from male population; Negative values show bias towards females; Transversal dimensions measured in centimeters; Body weight measured in kilograms.

(d)

\begin{tabular}{|c|c|c|c|c|c|c|c|c|c|c|c|c|c|c|c|c|}
\hline \multirow{3}{*}{ Sex } & \multirow{3}{*}{ Age } & \multirow{3}{*}{$\mathrm{N}$} & \multicolumn{14}{|c|}{ Circumferential Dimensions } \\
\hline & & & \multicolumn{2}{|c|}{$\begin{array}{c}\text { Chest } \\
\text { Circumference }\end{array}$} & \multicolumn{2}{|c|}{$\begin{array}{l}\text { Upper Arm } \\
\text { Circumference }\end{array}$} & \multicolumn{2}{|c|}{$\begin{array}{c}\text { Forearm } \\
\text { Circumference }\end{array}$} & \multicolumn{2}{|c|}{$\begin{array}{c}\text { Hip } \\
\text { Circumference }\end{array}$} & \multicolumn{2}{|c|}{$\begin{array}{c}\text { Waist } \\
\text { Circumference }\end{array}$} & \multicolumn{2}{|c|}{$\begin{array}{c}\text { Upper Leg } \\
\text { Circumference }\end{array}$} & \multicolumn{2}{|c|}{$\begin{array}{c}\text { Calf } \\
\text { Circumference }\end{array}$} \\
\hline & & & Mean & $\mathrm{SD}$ & Mean & SD & Mean & SD & Mean & $\mathrm{SD}$ & Mean & $\mathrm{SD}$ & Mean & SD & Mean & $\mathrm{SD}$ \\
\hline \multirow{7}{*}{ Male } & 7 & 30 & 61.6 & 4.2 & 18.1 & 2.1 & 17.9 & 1.8 & 65.7 & 5.3 & 55.7 & 5.1 & 37.2 & 3.6 & 25.4 & 2.5 \\
\hline & 8 & 35 & 64.4 & 4.8 & 19.4 & 1.9 & 19.1 & 1.5 & 69.1 & 5.2 & 59.1 & 4.9 & 39.7 & 4.1 & 26.9 & 1.9 \\
\hline & 10 & 42 & 72.0 & 7.9 & 21.1 & 2.8 & 20.5 & 2.0 & 75.4 & 8.3 & 65.2 & 9.5 & 43.0 & 6.7 & 28.9 & 3.1 \\
\hline & 11 & 59 & 74.3 & 7.6 & 22.1 & 3.1 & 20.6 & 2.0 & 81.1 & 8.6 & 67.1 & 8.7 & 48.0 & 6.7 & 31.0 & 3.3 \\
\hline & 12 & 49 & 76.7 & 7.0 & 22.9 & 3.2 & 21.8 & 2.0 & 81.1 & 9.2 & 67.3 & 7.6 & 47.7 & 8.8 & 31.1 & 3.4 \\
\hline & 13 & 63 & 78.8 & 7.9 & 23.7 & 3.2 & 22.4 & 2.2 & 86.6 & 8.4 & 69.6 & 7.5 & 51.0 & 8.6 & 33.3 & 3.8 \\
\hline & 14 & 58 & 80.6 & 7.2 & 24.0 & 2.4 & 23.2 & 1.9 & 86.0 & 7.4 & 69.1 & 5.1 & 48.4 & 5.3 & 33.3 & 2.9 \\
\hline \multirow{7}{*}{ Female } & 8 & 38 & 63.6 & 4.8 & 19.1 & 2.1 & 18.3 & 1.6 & 69.8 & 5.6 & 57.2 & 5.4 & 40.3 & 4.2 & 26.9 & 2.2 \\
\hline & 9 & 50 & 66.0 & 6.4 & 19.6 & 2.8 & 18.9 & 1.9 & 72.9 & 7.6 & 57.5 & 7.0 & 42.8 & 5.5 & 27.9 & 2.7 \\
\hline & 10 & 42 & 70.0 & 6.9 & 20.6 & 2.5 & 19.8 & 2.0 & 75.8 & 7.3 & 60.5 & 6.7 & 43.7 & 5.1 & 28.4 & 3.1 \\
\hline & 11 & 58 & 74.3 & 7.9 & 22.4 & 3.1 & 20.9 & 2.0 & 80.5 & 8.1 & 63.4 & 8.4 & 46.1 & 6.4 & 30.9 & 3.3 \\
\hline & 12 & 43 & 78.2 & 7.0 & 23.2 & 3.0 & 21.5 & 2.1 & 83.1 & 6.9 & 64.1 & 5.6 & 48.8 & 5.3 & 31.9 & 3.3 \\
\hline & 13 & 45 & 80.4 & 6.8 & 23.4 & 3.1 & 21.5 & 2.1 & 89.1 & 8.3 & 66.0 & 6.5 & 51.5 & 6.0 & 33.3 & 2.9 \\
\hline & 14 & 42 & 84.2 & 5.7 & 23.5 & 2.4 & 22.1 & 1.9 & 85.2 & 7.9 & 67.3 & 6.7 & 53.4 & 5.1 & 32.7 & 3.4 \\
\hline \multicolumn{3}{|c|}{$\begin{array}{l}\text { Average difference } \\
\text { between genders across } \\
\text { all age groups }\end{array}$} & ${ }^{\dagger}-0.1$ & $\dagger^{\dagger} 2.1$ & ${ }^{\dagger} 0.1$ & ${ }^{\dagger} 0.5$ & ${ }^{\dagger} 0.5$ & ${ }^{\dagger} 0.4$ & ${ }^{\dagger}-0.6$ & ${ }^{\dagger} 1.2$ & $\dagger 3.1$ & ${ }^{\dagger} 1.4$ & ${ }^{\dagger}-0.9$ & $\dagger^{\dagger} .0$ & ${ }^{\dagger} 0.1$ & ${ }^{\dagger} 0.5$ \\
\hline
\end{tabular}

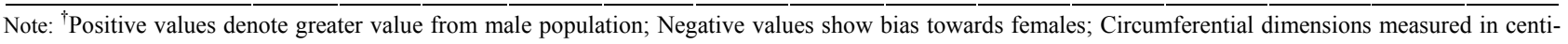
metres. 
Table 2.

(a) Correlation values between torso endurance scores and anthropometric measurements for boys; (b) Correlation values between torso endurance scores and anthropometric measurements for girls.

(a)

\begin{tabular}{|c|c|c|c|c|c|c|c|c|c|}
\hline & \multirow[b]{2}{*}{ Boys } & \multicolumn{5}{|c|}{ Longitudinal Dimensions } & \multicolumn{3}{|c|}{ Transversal Dimensions } \\
\hline & & Body Height & Sitting Height & Leg Length & Arm Length & $\begin{array}{l}\text { Upper Arm } \\
\text { Length }\end{array}$ & $\begin{array}{l}\text { Shoulder } \\
\text { Diameter }\end{array}$ & $\begin{array}{c}\text { Pelvis } \\
\text { Diameter }\end{array}$ & $\begin{array}{c}\text { Knee } \\
\text { Diameter }\end{array}$ \\
\hline \multirow{2}{*}{ BET } & $\mathrm{R}$ & $0.27^{* *}$ & $0.25^{* *}$ & $0.26^{* *}$ & $0.28^{* *}$ & $0.27^{* *}$ & $0.26^{* *}$ & $0.23^{* *}$ & 0.09 \\
\hline & alpha & $<0.001$ & $<0.001$ & $<0.001$ & $<0.001$ & $<0.001$ & $<0.001$ & $<0.001$ & 0.09 \\
\hline \multirow{2}{*}{ FET } & $\mathrm{R}$ & 0.09 & 0.10 & 0.09 & 0.08 & 0.08 & 0.06 & 0.03 & -0.04 \\
\hline & alpha & 0.08 & 0.06 & 0.07 & 0.12 & 0.13 & 0.23 & 0.55 & 0.40 \\
\hline \multirow{2}{*}{ LS } & $\mathrm{R}$ & $0.16^{* *}$ & $0.18^{* *}$ & $0.13^{* *}$ & $0.15^{* *}$ & $0.16^{* *}$ & $0.15^{* *}$ & $0.10^{*}$ & -0.05 \\
\hline & alpha & $<0.001$ & $<0.001$ & 0.01 & $<0.001$ & $<0.001$ & $<0.001$ & 0.04 & 0.35 \\
\hline \multirow{2}{*}{ RS } & $\mathrm{R}$ & $0.13^{* *}$ & $0.14^{* *}$ & $0.11^{*}$ & $0.13^{*}$ & $0.10^{*}$ & $0.12^{*}$ & 0.05 & -0.09 \\
\hline & alpha & $<0.001$ & $<0.001$ & 0.03 & 0.01 & 0.04 & 0.02 & 0.29 & 0.09 \\
\hline \multicolumn{10}{|c|}{ Circumferential Dimensions } \\
\hline \multicolumn{2}{|r|}{ Boys } & $\begin{array}{c}\text { Chest } \\
\text { Circumference }\end{array}$ & $\begin{array}{l}\text { Upper Arm } \\
\text { Circumference }\end{array}$ & $\begin{array}{c}\text { Forearm } \\
\text { Circumference }\end{array}$ & $\begin{array}{c}\text { Hip } \\
\text { Circumference }\end{array}$ & $\begin{array}{c}\text { Waist } \\
\text { Circumference }\end{array}$ & $\begin{array}{c}\text { Upper Leg } \\
\text { Circumference }\end{array}$ & $\begin{array}{c}\text { Calf } \\
\text { Circumference }\end{array}$ & Body Weight \\
\hline \multirow{2}{*}{ BET } & $\mathrm{R}$ & $0.12^{*}$ & 0.04 & 0.06 & $0.11^{*}$ & 0.05 & 0.06 & $0.11^{*}$ & $0.16^{* *}$ \\
\hline & alpha & 0.01 & 0.46 & 0.24 & 0.03 & 0.37 & 0.25 & 0.04 & $<0.001$ \\
\hline \multirow{2}{*}{ FET } & $\mathrm{R}$ & 0.00 & -0.03 & -0.04 & 0.00 & -0.03 & -0.01 & -0.03 & 0.01 \\
\hline & alpha & 0.98 & 0.51 & 0.47 & 0.96 & 0.56 & 0.80 & 0.57 & 0.77 \\
\hline \multirow{2}{*}{ LS } & $\mathrm{R}$ & 0.05 & -0.04 & 0.04 & -0.01 & -0.07 & -0.06 & 0.02 & 0.03 \\
\hline & alpha & 0.37 & 0.40 & 0.45 & 0.87 & 0.15 & 0.23 & 0.76 & 0.50 \\
\hline \multirow{2}{*}{ RS } & $\mathrm{R}$ & -0.01 & $-0.10^{*}$ & -0.03 & -0.05 & $-0.12^{*}$ & -0.09 & -0.04 & -0.02 \\
\hline & alpha & 0.91 & 0.05 & 0.60 & 0.35 & 0.01 & 0.08 & 0.42 & 0.74 \\
\hline
\end{tabular}

Note: Statistically significant $\left({ }^{*} p<0.05,{ }^{* *} p<0.01\right)$; BET—back extension test (Biering-Sorensen); FET—flexor endurance test (sit-up posture); LS—left side bridge; RS-right side bridge.

(b)

\begin{tabular}{|c|c|c|c|c|c|c|c|c|c|}
\hline & & \multicolumn{5}{|c|}{ Longitudinal Dimensions } & \multicolumn{3}{|c|}{ Transversal Dimensions } \\
\hline \multicolumn{2}{|c|}{ Girls } & Body Height & Sitting Height & Leg Length & Arm Length & $\begin{array}{l}\text { Upper Arm } \\
\text { Length }\end{array}$ & $\begin{array}{l}\text { Shoulder } \\
\text { Diameter }\end{array}$ & $\begin{array}{c}\text { Pelvis } \\
\text { Diameter }\end{array}$ & $\begin{array}{c}\text { Knee } \\
\text { Diameter }\end{array}$ \\
\hline \multirow{2}{*}{ BET } & $\mathrm{R}$ & $0.35^{* *}$ & $0.32^{* *}$ & $0.37^{* *}$ & $0.35^{* *}$ & $0.34^{* *}$ & $0.30^{* *}$ & $0.26^{* *}$ & $0.17^{* *}$ \\
\hline & alpha & $<0.001$ & $<0.001$ & $<0.001$ & $<0.001$ & $<0.001$ & $<0.001$ & $<0.001$ & 0.001 \\
\hline \multirow{2}{*}{ FET } & $\mathrm{R}$ & $0.16^{* *}$ & $0.14^{* *}$ & $0.17^{* *}$ & $0.13^{*}$ & $0.15^{* *}$ & 0.101 & 0.090 & 0.048 \\
\hline & alpha & 0.002 & 0.007 & 0.002 & 0.012 & 0.004 & 0.055 & 0.088 & 0.363 \\
\hline \multirow{2}{*}{ LS } & $\mathrm{R}$ & $0.19^{* *}$ & $0.20^{* *}$ & $0.15^{* *}$ & $0.18^{* *}$ & $0.18^{* *}$ & $0.12^{*}$ & $0.12^{*}$ & -0.049 \\
\hline & alpha & $<0.001$ & $<0.001$ & 0.004 & 0.001 & 0.001 & 0.019 & 0.026 & 0.357 \\
\hline \multirow{2}{*}{ RS } & $\mathrm{R}$ & $0.17^{* *}$ & $0.19^{* *}$ & $0.14^{* *}$ & $0.17^{* *}$ & $0.16^{* *}$ & 0.092 & $0.12^{*}$ & -0.063 \\
\hline & alpha & 0.001 & $<0.001$ & 0.008 & 0.001 & 0.003 & 0.081 & 0.028 & 0.237 \\
\hline \multicolumn{10}{|c|}{ Circumferential Dimensions } \\
\hline \multicolumn{2}{|c|}{ Girls } & $\begin{array}{c}\text { Chest } \\
\text { Circumference }\end{array}$ & $\begin{array}{c}\text { Upper Arm } \\
\text { Circumference }\end{array}$ & $\begin{array}{c}\text { Forearm } \\
\text { Circumference }\end{array}$ & $\begin{array}{c}\text { Hip } \\
\text { Circumference }\end{array}$ & $\begin{array}{c}\text { Waist } \\
\text { Circumference }\end{array}$ & $\begin{array}{c}\text { Upper Leg } \\
\text { Circumference }\end{array}$ & $\begin{array}{l}\text { Calf } \\
\text { ircumference }\end{array}$ & Body Weight \\
\hline \multirow{2}{*}{ BET } & $\mathrm{R}$ & $0.24^{* *}$ & $0.17^{* *}$ & $0.22^{* *}$ & $0.26^{* *}$ & $0.17^{* *}$ & $0.24^{* *}$ & $0.22^{* *}$ & $0.27^{* *}$ \\
\hline & alpha & $<0.001$ & 0.002 & $<0.001$ & $<0.001$ & 0.002 & $<0.001$ & $<0.001$ & $<0.001$ \\
\hline \multirow{2}{*}{ FET } & $\mathrm{R}$ & 0.064 & 0.065 & 0.042 & 0.097 & 0.040 & 0.086 & 0.062 & $0.11^{*}$ \\
\hline & alpha & 0.229 & 0.217 & 0.430 & 0.066 & 0.448 & 0.105 & 0.245 & 0.043 \\
\hline \multirow{2}{*}{ LS } & $\mathrm{R}$ & 0.071 & -0.013 & 0.052 & 0.018 & -0.061 & 0.013 & 0.033 & 0.072 \\
\hline & alpha & 0.182 & 0.800 & 0.322 & 0.739 & 0.252 & 0.809 & 0.527 & 0.176 \\
\hline \multirow{2}{*}{ RS } & $\mathrm{R}$ & 0.061 & -0.015 & 0.040 & 0.018 & -0.049 & $<0.001$ & 0.040 & 0.062 \\
\hline & alpha & 0.250 & 0.779 & 0.449 & 0.728 & 0.351 & 0.996 & 0.449 & 0.238 \\
\hline
\end{tabular}

Note: Statistically significant $\left({ }^{*} p<0.05,{ }^{* *} p<0.01\right)$; BET—back extension test (Biering-Sorensen); FET—flexor endurance test (sit-up posture); LS—left side bridge; RS—right side bridge. 
Table 3.

(a) Correlation values for boys of the 4 most correlated anthropometric variables with the 4 torso endurance tests; (b) Correlation values for girls of the 4 most correlated anthropometric variables with the 4 torso endurance tests.

(a)

\begin{tabular}{|c|c|c|c|c|c|c|c|c|c|c|c|c|c|c|c|c|c|}
\hline & \multicolumn{9}{|c|}{ Knee Diameter } & \multicolumn{8}{|c|}{ Forearm Circumference } \\
\hline & Age & 7 & 8 & 9 & 10 & 11 & 12 & 13 & 14 & 7 & 8 & 9 & 10 & 11 & 12 & 13 & 14 \\
\hline \multirow{2}{*}{ BET } & $\mathrm{R}$ & -0.21 & -0.16 & 0.00 & $-0.32^{*}$ & -0.25 & -0.03 & $-0.25^{*}$ & 0.13 & -0.25 & -0.12 & -0.25 & $-0.30^{*}$ & $-0.27^{*}$ & $-0.35^{*}$ & $-0.28^{*}$ & 0.17 \\
\hline & Sig. & 0.27 & 0.35 & 0.98 & 0.04 & 0.06 & 0.82 & 0.05 & 0.34 & 0.18 & 0.48 & 0.05 & 0.05 & 0.04 & 0.02 & 0.03 & 0.20 \\
\hline \multirow{2}{*}{ FET } & $\mathrm{R}$ & 0.14 & -0.24 & 0.12 & $-0.39^{*}$ & -0.11 & $-0.32^{*}$ & $-0.28^{*}$ & -0.16 & -0.01 & -0.23 & -0.03 & $-0.39^{*}$ & $-0.27^{*}$ & -0.25 & -0.24 & $-0.27^{*}$ \\
\hline & Sig. & 0.47 & 0.17 & 0.35 & 0.01 & 0.39 & 0.03 & 0.03 & 0.24 & 0.96 & 0.18 & 0.82 & 0.01 & 0.04 & 0.08 & 0.06 & 0.04 \\
\hline \multirow{2}{*}{ LS } & $\mathrm{R}$ & -0.28 & -0.19 & -0.03 & $-0.31^{*}$ & $-0.33^{* *}$ & -0.12 & $-0.27^{*}$ & $-0.26^{*}$ & -0.04 & -0.02 & -0.21 & $-0.44^{* *}$ & -0.22 & -0.10 & -0.12 & -0.07 \\
\hline & Sig. & 0.14 & 0.27 & 0.81 & 0.04 & 0.01 & 0.41 & 0.03 & 0.05 & 0.82 & 0.91 & 0.11 & 0.00 & 0.10 & 0.48 & 0.33 & 0.62 \\
\hline \multirow{4}{*}{$\mathrm{RS}$} & $\mathrm{R}$ & -0.07 & $-0.39^{*}$ & -0.07 & $-0.51^{* *}$ & $-0.53^{* *}$ & -0.23 & $-0.41^{* *}$ & -0.14 & 0.06 & -0.11 & $-0.28^{*}$ & $-0.50^{* *}$ & $-0.53^{* *}$ & -0.19 & $-0.28^{*}$ & 0.06 \\
\hline & Sig. & 0.70 & 0.02 & 0.58 & 0.00 & 0.00 & 0.12 & 0.00 & 0.29 & 0.74 & 0.54 & 0.03 & 0.00 & 0.00 & 0.20 & 0.03 & 0.64 \\
\hline & & \multicolumn{8}{|c|}{ Upper Leg Circumference } & \multicolumn{8}{|c|}{ Body Weight } \\
\hline & Age & 7 & 8 & 9 & 10 & 11 & 12 & 13 & 14 & 7 & 8 & 9 & 10 & 11 & 12 & 13 & 14 \\
\hline \multirow{2}{*}{ BET } & $\mathrm{R}$ & -0.03 & -0.09 & -0.18 & $-0.31^{*}$ & $-0.29^{*}$ & -0.12 & $-0.31^{*}$ & 0.15 & -0.20 & -0.14 & -0.09 & $-0.32^{*}$ & -0.22 & -0.22 & $-0.26^{*}$ & 0.19 \\
\hline & Sig. & 0.88 & 0.63 & 0.18 & 0.04 & 0.03 & 0.40 & 0.01 & 0.26 & 0.28 & 0.44 & 0.51 & 0.04 & 0.09 & 0.12 & 0.04 & 0.16 \\
\hline \multirow{2}{*}{ FET } & $\mathrm{R}$ & -0.11 & -0.20 & 0.06 & $-0.42^{* *}$ & -0.14 & -0.25 & -0.12 & -0.24 & 0.01 & -0.22 & 0.04 & $-0.46^{* *}$ & -0.17 & $-0.34^{*}$ & -0.15 & $-0.27^{*}$ \\
\hline & Sig. & 0.56 & 0.24 & 0.64 & 0.01 & 0.28 & 0.09 & 0.36 & 0.07 & 0.98 & 0.21 & 0.75 & 0.00 & 0.21 & 0.02 & 0.23 & 0.04 \\
\hline \multirow{2}{*}{ LS } & $\mathrm{R}$ & 0.11 & -0.08 & $-0.26^{*}$ & $-0.54^{* *}$ & $-0.34^{* *}$ & -0.14 & -0.18 & $-0.26^{*}$ & -0.11 & -0.06 & -0.21 & $-0.53^{* *}$ & $-0.36^{* *}$ & -0.20 & -0.22 & -0.23 \\
\hline & Sig. & 0.57 & 0.66 & 0.05 & 0.00 & 0.01 & 0.34 & 0.16 & 0.05 & 0.56 & 0.72 & 0.12 & 0.00 & 0.01 & 0.18 & 0.09 & 0.09 \\
\hline \multirow{2}{*}{ RS } & $\mathrm{R}$ & 0.24 & -0.20 & $-0.38^{* *}$ & $-0.68^{* *}$ & $-0.51^{* *}$ & -0.20 & $-0.30^{*}$ & 0.04 & 0.05 & -0.17 & $-0.30^{*}$ & $-0.70^{* *}$ & $-0.55^{* *}$ & $-0.33^{*}$ & $-0.40^{* *}$ & -0.08 \\
\hline & Sig. & 0.21 & 0.25 & 0.00 & 0.00 & 0.00 & 0.17 & 0.02 & 0.78 & 0.80 & 0.32 & 0.02 & 0.00 & 0.00 & 0.02 & 0.00 & 0.54 \\
\hline
\end{tabular}

Note: Significant correlations ( ${ }^{*} p<0.05,{ }^{* *} p<0.01$ ); BET—back extension test (Biering-Sorensen); FET—flexor endurance test (sit-up posture); LS—left side bridge; RS_right side bridge.

(b)

\begin{tabular}{|c|c|c|c|c|c|c|c|c|c|c|c|c|c|c|c|c|c|}
\hline & \multicolumn{9}{|c|}{ Body Height } & \multicolumn{8}{|c|}{ Sitting Height } \\
\hline & Age & 7 & 8 & 9 & 10 & 11 & 12 & 13 & 14 & 7 & 8 & 9 & 10 & 11 & 12 & 13 & 14 \\
\hline \multirow{2}{*}{ BET } & $\mathrm{R}$ & $0.35^{*}$ & $0.38^{*}$ & -0.12 & 0.21 & 0.08 & 0.05 & -0.04 & -0.16 & 0.29 & $0.40^{*}$ & -0.10 & 0.12 & 0.03 & -0.19 & -0.13 & -0.19 \\
\hline & Sig. & 0.02 & 0.02 & 0.42 & 0.19 & 0.53 & 0.77 & 0.80 & 0.30 & 0.06 & 0.01 & 0.47 & 0.47 & 0.85 & 0.23 & 0.40 & 0.23 \\
\hline \multirow{2}{*}{ FET } & $\mathrm{R}$ & $0.47^{* *}$ & 0.15 & -0.27 & -0.13 & 0.06 & 0.02 & -0.15 & 0.13 & $0.413^{* *}$ & 0.17 & $-0.296^{*}$ & -0.15 & -0.01 & -0.08 & -0.23 & 0.10 \\
\hline & Sig. & 0.00 & 0.37 & 0.05 & 0.40 & 0.66 & 0.91 & 0.32 & 0.41 & 0.01 & 0.29 & 0.04 & 0.34 & 0.97 & 0.62 & 0.13 & 0.52 \\
\hline \multirow{2}{*}{ LS } & $\mathrm{R}$ & -0.10 & 0.07 & $-0.35^{*}$ & 0.04 & -0.03 & 0.07 & -0.12 & -0.27 & 0.08 & 0.17 & $-0.34^{*}$ & 0.00 & 0.03 & -0.03 & -0.06 & $-0.44^{* *}$ \\
\hline & Sig. & 0.53 & 0.69 & 0.01 & 0.81 & 0.82 & 0.64 & 0.45 & 0.09 & 0.62 & 0.31 & 0.02 & 0.99 & 0.84 & 0.86 & 0.69 & 0.00 \\
\hline \multirow{4}{*}{ RS } & $\mathrm{R}$ & -0.06 & $0.38^{*}$ & $-0.44^{* *}$ & -0.02 & 0.00 & 0.10 & 0.00 & $-0.32^{*}$ & 0.08 & $0.50^{* *}$ & $-0.38^{* *}$ & -0.05 & 0.07 & 0.14 & 0.01 & $-0.43^{* *}$ \\
\hline & Sig. & 0.72 & 0.02 & 0.00 & 0.89 & 0.97 & 0.53 & 0.98 & 0.04 & 0.64 & 0.00 & 0.01 & 0.77 & 0.62 & 0.36 & 0.92 & 0.00 \\
\hline & & \multicolumn{8}{|c|}{ Leg Length } & \multicolumn{8}{|c|}{ Pelvic Diameter } \\
\hline & Age & 7 & 8 & 9 & 10 & 11 & 12 & 13 & 14 & 7 & 8 & 9 & 10 & 11 & 12 & 13 & 14 \\
\hline \multirow{2}{*}{ BET } & $\mathrm{R}$ & $0.35^{*}$ & $0.34^{*}$ & -0.11 & 0.22 & 0.09 & 0.29 & -0.02 & -0.16 & 0.21 & 0.06 & $-0.28^{*}$ & 0.17 & -0.03 & -0.15 & -0.17 & $-0.34^{*}$ \\
\hline & Sig. & 0.03 & 0.04 & 0.43 & 0.15 & 0.48 & 0.06 & 0.88 & 0.32 & 0.18 & 0.70 & 0.05 & 0.29 & 0.84 & 0.35 & 0.28 & 0.03 \\
\hline \multirow{2}{*}{ FET } & $\mathrm{R}$ & $0.41^{* *}$ & 0.10 & $-0.31^{*}$ & -0.04 & -0.01 & 0.21 & -0.09 & 0.05 & $0.38^{*}$ & -0.05 & -0.17 & -0.14 & $-0.36^{* *}$ & 0.06 & -0.19 & -0.07 \\
\hline & Sig. & 0.01 & 0.54 & 0.03 & 0.81 & 0.97 & 0.18 & 0.57 & 0.76 & 0.02 & 0.75 & 0.25 & 0.36 & 0.01 & 0.71 & 0.22 & 0.68 \\
\hline \multirow{2}{*}{ LS } & $\mathrm{R}$ & -0.17 & -0.06 & $-0.31^{*}$ & 0.04 & -0.19 & 0.03 & -0.17 & $-0.33^{*}$ & 0.03 & -0.04 & $-0.54^{* *}$ & -0.12 & -0.20 & -0.08 & -0.02 & -0.26 \\
\hline & Sig. & 0.29 & 0.70 & 0.03 & 0.79 & 0.16 & 0.85 & 0.27 & 0.03 & 0.88 & 0.81 & 0.00 & 0.43 & 0.14 & 0.63 & 0.89 & 0.10 \\
\hline \multirow{2}{*}{ RS } & $\mathrm{R}$ & -0.11 & 0.19 & $-0.43^{* *}$ & -0.03 & -0.10 & 0.02 & -0.15 & $-0.40^{* *}$ & -0.04 & 0.14 & $-0.40^{* *}$ & -0.12 & -0.16 & 0.27 & -0.10 & $-0.32^{*}$ \\
\hline & Sig. & 0.48 & 0.26 & 0.00 & 0.85 & 0.48 & 0.92 & 0.32 & 0.01 & 0.81 & 0.42 & 0.00 & 0.47 & 0.22 & 0.08 & 0.53 & 0.04 \\
\hline
\end{tabular}

Note: Significant correlations $\left({ }^{*} p<0.05,{ }^{* *} p<0.01\right)$; BET—back extension test (Biering-Sorensen); FET—flexor endurance test (sit-up posture); LS—left side bridge; RS - right side bridges. 


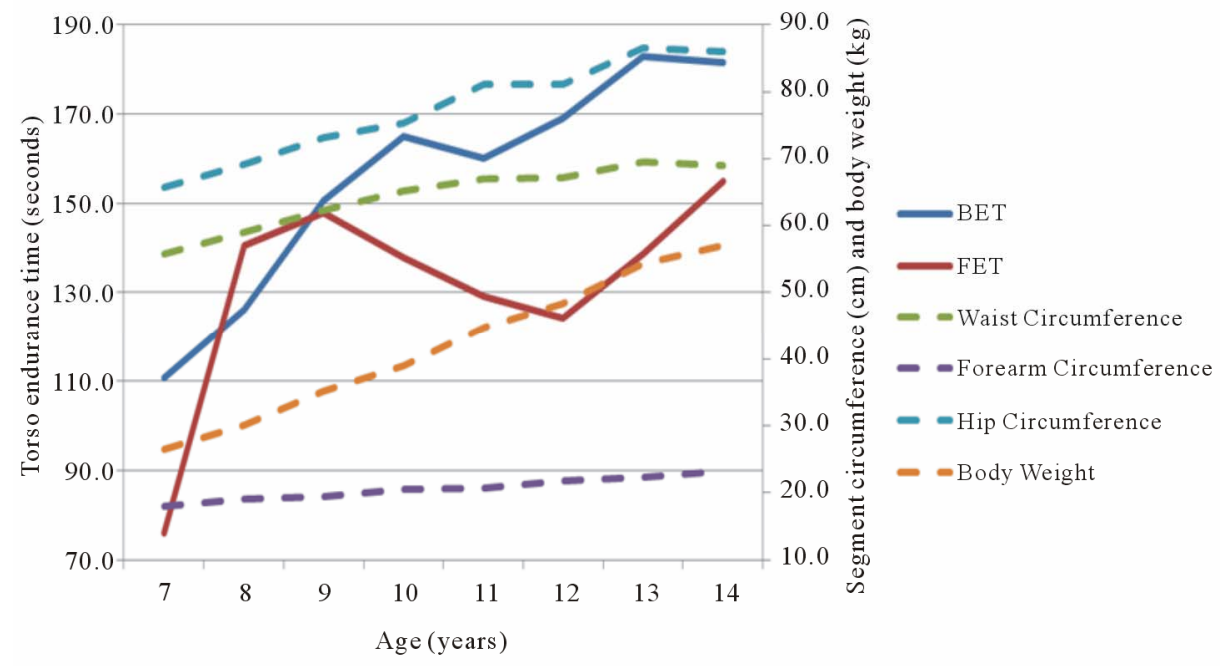

Figure 2.

Torso extensor and flexor endurance and anthropometric changes with age in boys illustrating progressive increase in anthropometric variables with age while endurance scores showed inflection points.

performance for these tests occurred at age 8 and between ages 11 and 12 before increases were seen. Figures 2 and $\mathbf{3}$ show the non-linear changes in torso extensor/flexor endurance and lateral torso endurance, respectively, along with the year by year systematic increase in the 4 anthropometric variables that were most correlated with the torso endurance tests (knee diameter, forearm circumference, upper leg circumference and body weight).

\section{Girls}

Similar to the boys, correlations were strongest between BET and longitudinal measures for the girls when taken across all ages; however, the girls showed higher correlations than the boys. The girls followed the same pattern of significant, but weak, correlations between FET, LS and RS and anthropometrics (Table 2(b)).

While transversal and circumferential dimensions and body weight were most correlated with torso endurance scores within each age group for the boys, the longitudinal dimensions dominated these correlations in the girls. Body height, followed by sitting height, leg length and pelvic diameter were the highest correlated anthropometric measures with BET, FET, RS and LS (Table 3(b)).

There were also similarities in the changes in anthropometric variables and torso endurance scores between the boys and girls; however, there were differences in the torso endurance inflection peaks and valleys. BET scores demonstrated the first peak at age 10 before dropping at age 11 and increasing to age 12, leveling out for ages 13 and 14. FET times increased a year later in girls than they did in boys and did not increase as dramatically; an increase in scores of $74 \%$ was seen from age 8 to 9. There was a drop from age 9 to 10 and then even more substantial from 10 to 11 before an increase was seen through age 13. Lateral torso endurance scores in girls changed most similarly to boys; there was, however, an initial peak in RS and LS at age 10. Figures $\mathbf{4}$ and $\mathbf{5}$ show these changes along with the linear changes in the anthropometric variables that were most correlated with the 4 torso endurance tests (body height, sitting height, leg length and pelvic diameter).

\section{Boys vs. Girls}

Girls had better BET scores than boys with an average difference of 23.9s across all age groups. Though boys had higher scores in the other 3 endurance tests, the greatest difference between the genders was only 6.1s (LS) (Table 1).

The Kruskal-Wallis test determined there was a significant effect of gender on BET $(\mathrm{H}(1)=17.675, p<0.001)$ and RS $(\mathrm{H}(1)=9.366, p=0.002)$, but not FET $(p=0.216)$ and LS $(p=$ $0.111)$ scores. The Mann-Whitney U statistic showed that girls had significantly greater BET scores than the boys at ages $9(\mathrm{U}=$ $929.5, p=0.001)$ and $12(\mathrm{U}=628, p=0.001)$. There were no significant differences between the genders in RS scores $(p>$ $0.047)$.

\section{Discussion}

The most surprising result of this study was that torso endurance scores seem to change independently of age. When all individuals were considered together regardless of age, the only pattern that was detected existed between longitudinal dimensions and endurance times. Though significant correlations existed between several anthropometric variables and torso endurance times, the magnitudes were very low. Despite the wide range of body measurements taken in multiple dimensions, there were no notable trends in the correlations. There are other factors in children, less related to body size, which must influence torso muscle endurance. Perhaps hormonal changes associated with puberty or other variables that affect one's effort during physical exertions may have more of an impact on torso endurance testing than the length of their legs. Thus, the first hypothesis must be rejected that anthropometric features influence endurance times. The second hypothesis suggesting boys and girls differ both in endurance profiles and their links to anthropometric variables is accepted.

There are divided opinions regarding gender differences for back muscle endurance in the literature. Some authors found significantly higher endurance scores in females (Kankaanpää 

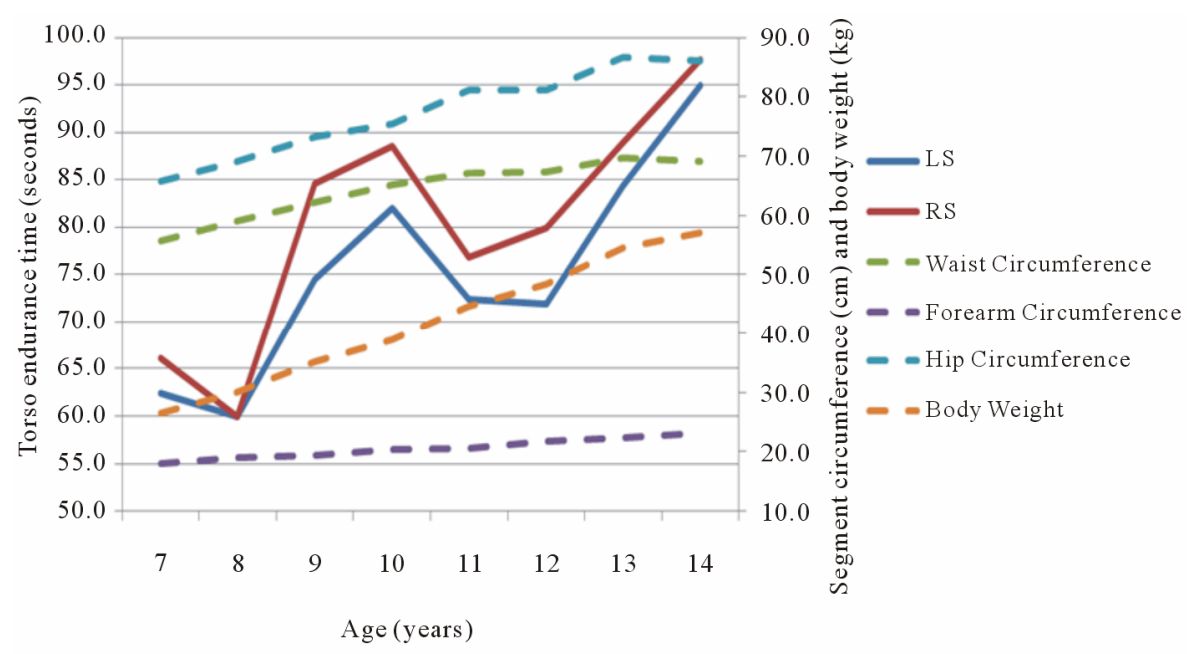

Figure 3.

Lateraltorso endurance and anthropometric changes with age in boys illustrating progressive increase in anthropometric variables with age while endurance scores showed inflection points.

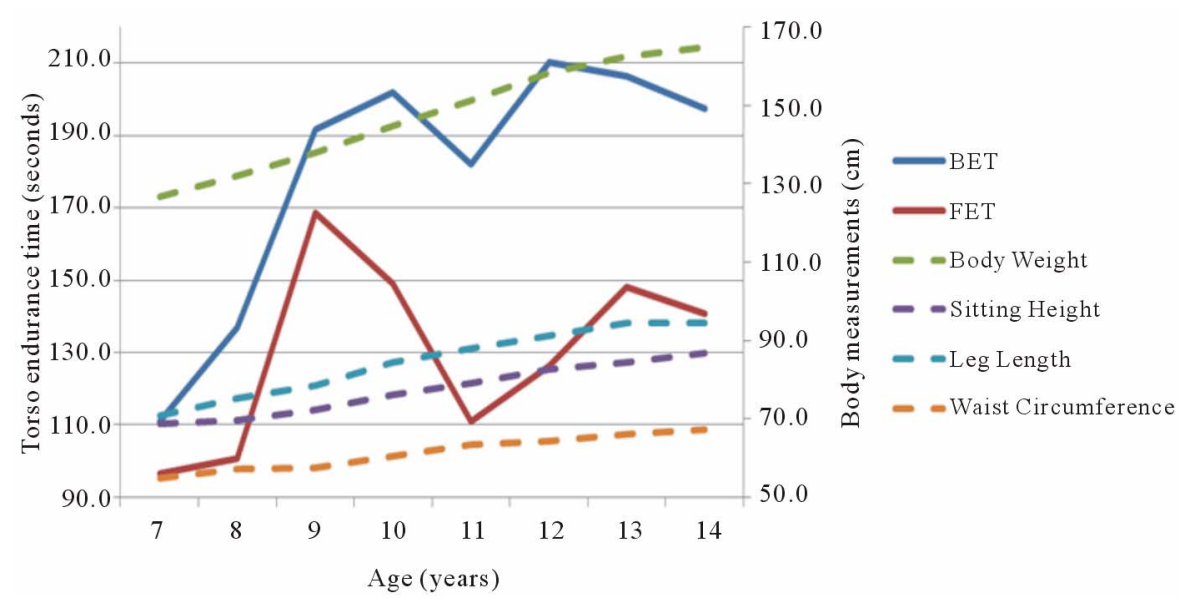

Figure 4 .

Torso extensor and flexor endurance and anthropometric changes with age in girls illustrating progressive increase in anthropometric variables with age while endurance scores showed inflection points.

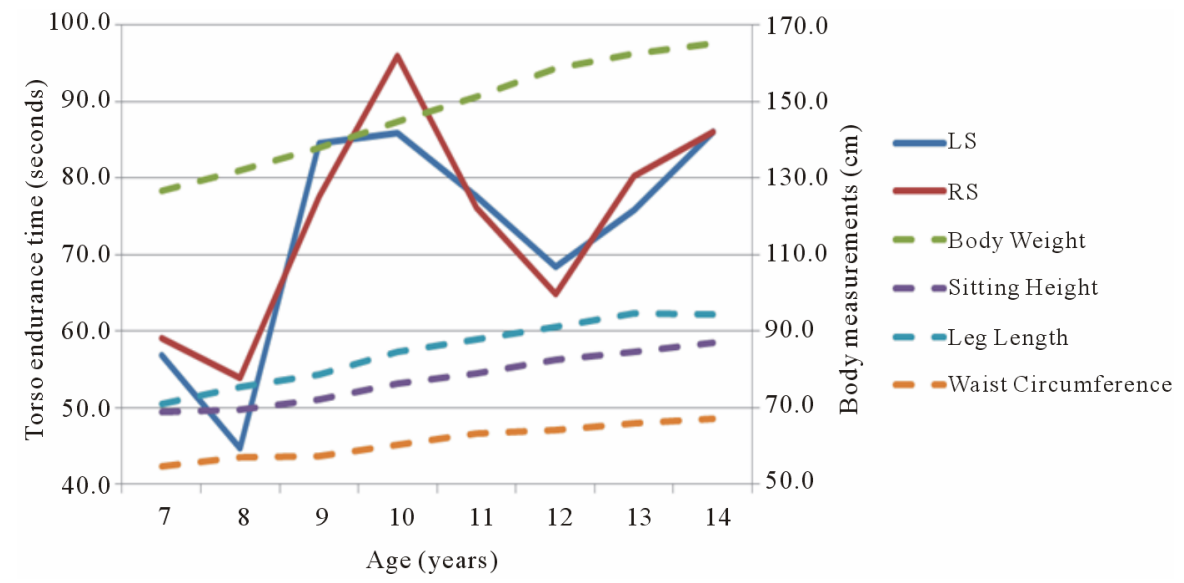

Figure 5.

Lateraltorso endurance and anthropometric changes with age in girls illustrating progressive increase in anthropometric variables with age while endurance scores showed inflection points. 
et al., 1998), while other studies claim the opposite (Alaranta et al., 1994). Another factor related to gender that contributes to back muscle endurance may be differences in body segment proportions, as suggested by the data from the current study. Tichauer et al. (1978) reported that females had longer torsos and shorter legs than males, suggesting they can achieve better endurance times in back endurance tests because of it; however, similar to the findings of Xiao et al. (2005), the results from the current study failed to support this notion with any clear pattern between segment length and torso endurance.

One of the problems in this controversy is that some back muscle endurance scores were achieved in healthy populations while the others were collected from low back pain (LBP) subjects. Our study was limited to healthy children aged 7 to 14 and results showed that girls are able to maintain longer back extension. One of the reasons for this may be due to greater lumbar lordosis in girls, allowing for higher mechanical advantage of the spinal erector muscles, as suggested by Tviet et al. (1994) and McIntosh et al. (1993). The different geometry of the female torso from the male torso (Marras et al., 2001), as well as a presence of a greater number of type I fibers in lumbar region (Mannion et al., 1997) could potentially influence spine loading. Some limitations have to be considered for interpreting the data of this study. We have found no data sets with which to directly compare these results. The literature focuses on correlations between BMI, body height and body weight with torso endurance or grip strength. Another limitation arises from the fact that results were obtained from Serbian children from one elementary school aged $7-14$. Personal factors (motivation, for example) may have influenced performance on torso endurance tests, which may complicate the interpretation of the results. In an effort to protect against this, however, the children were encouraged during all torso endurance testing.

\section{Conclusion}

Boys and girls aged 7 to 14 have different relationships between torso muscle endurance scores and anthropometric measures. However anthropometric variables appear to have little influence on torso endurance and appear to be influenced by other factors that were not measured in this study. This eventually may lead to gender specific prevention and management of LBP and exercise program development.

\section{REFERENCES}

Alaranta, H., Hurri, H., Heliovaara, M., Soukka, A., \& Harju, R. (1994). Non-dynamometric trunk performance tests: Reliability and normative data. Scandinavian Journal of Rehabilitation Medicine, 26, 211-215.

Biering-Sorensen, F. (1984). Physical measurements as physical risk indicators for low back trouble over a one-year period. Spine, 9, 106-119.

Dejanovic, A. (2006). Relacije antropometrijskih karakteristika i izometrijskog mišićnog potencijala u lumbalnoj i abdominalnoj regiji kod dece (Relations of anthropometric characteristics and isometric muscular potential in lumbar and abdominal region in children). Novi Sad: University of Novi Pazar.

Dejanovic, A., Harvey, E., \& McGill, S. (2012). Changes in torso muscle endurance profiles in children aged 7 to 14 years: Reference values. Archives of Physical Medicine and Rehabilitation, 93, 2295-2301.

Docherty, D., \& Gaul, C. A. (1991). Relationship of body size, phy- sique, and composition to physical performance in young boys and girls. International Journal of Sports Medicine, 12, 525-532. doi: $10.1055 / \mathrm{s}-2007-1024728$

Evans, K., Refshauge, K., \& Adams, R. (2007). Trunk muscle endurance tests: Reliability, and gender differences in athletes. Journal of Science and Medicine in Sport, 10, 447-455. doi:10.1016/j.jsams.2006.09.003

Everett, P. W., \& Sills, F. W. (1952). The relationship of grip strength to stature, somatotype components and anthropometric measurements of the hand. Research Quarterly, 23, 161-166.

Gabbard, C. P., \& Patterson, P. E. (1980). Relationship and comparison of selected anthropometric measures to muscular endurance and strength in children aged 3 - 5 years. Annals of Human Biology, 7, 583-586. doi:10.1080/03014468000004701

Johnson, B. (1925). Mental growth of children in relation to the rate of the growth in bodily development. A report of the Bureau of educational experiments. New York, NY: Dalton.

Jürimäe, T., \& Jürimäe, J. (2000). Growth, physical activity, and motor development in prepubertal children. Boca Raton, FL: CRC Press.

Kankaanpää, M., Laaksonen, D., Taimela, S., Kokko, S. M., Airaksinen, O., \& Hänninen, O. (1998). Age, sex, and body mass index as determinants of back and hip extensor fatigue in the isometric Sorensen back endurance test. Archives of Physical Medicine and Rehabilitation, 79, 1069-1075. doi:10.1016/S0003-9993(98)90173-3

Lohman, T. G., Roche, A. F., \& Martorell, R. (1988). Anthropometric standardization reference manual. Chicago, IL: Human Kinetics Books.

Mannion, A. F., Dumas, G. A., Cooper, R. G., Espinosa, F. J., Faris, M. W., \& Setevenson, J. M. (1997). Muscle fiber size and type distribution in thoracic and lumbar regions of erector spinae in healthy subjects with no low back pain: Normal values and gender differences. Journal of Anatomy, 190, 505-513. doi:10.1046/j.1469-7580.1997.19040505.x

Marras, W. S., Davis, K. G., Ferguson, S. A., Lucas, B., R., \& Gupta, P. (2001). Spine loading characteristics of patients with low back pain compared with asymptomatic individuals. Spine, 26, 2566-2574. doi:10.1097/00007632-200112010-00009

McGill, S. M., Childs, A., \& Liebenson, C. (1999). Endurance times for low back stabilization exercises: Clinical targets for testing and training from a normal database. Archives of Physical Medicine and Rehabilitation, 80, 941-944. doi:10.1016/S0003-9993(99)90087-4

McIntosh, J. E., Bogduk, N., \& Pearcy, M. J. (1993). The effects of flexion on the geometry and actions of the lumbar erector spine. Spine, 18, 884-893. doi:10.1097/00007632-199306000-00013

Metheny, E. (1941). The present status of strength testing for children of elementary school and preschool age. Research Quarterly, 12, 115-130.

Rarick, L. G., \& Dobbins, D. A. (1975). Basic components in the motor performance of children 6 - 9 years of age. Medicine and Science in Sports, 7, 105-110. doi:10.1249/00005768-197500720-00017

Salminen, J., Maki, P., Oksanen, A., \& Pentti, J. (1992). Spinal mobility and trunk muscle strength in 15-year-old schoolchildren with and without low-back pain. Spine, 17, 405-411. doi:10.1097/00007632-199204000-00005

Slaughter, M. H., Lohman, T. G., \& Misner, J. E. (1977). Relationship of somatotype and body composition to physical performance in 7to 12-year-old boys. Research Quarterly, 48, 159-168.

Tichauer, E. R. (1978). The biomechanical basis of ergonomics: Anatomy applied to the design of work stations. New York, NY: John Wiley and Sons.

Tveit, P., Daggfeldt, K., Hetland, S., \& Thorstensson, A. (1994). Erector spinae lever arm length variations with changes in spinal curvature. Spine, 19, 199-204. doi:10.1097/00007632-199401001-00015

Xiao, G., Lei, L., Demspey, P. G., Lu, B., \& Liang, Y. (2005). Isometric muscle strength and anthropometric characteristics of a Chinese sample. International Journal of Industrial Ergonomics, 35, 674-679. doi:10.1016/j.ergon.2005.02.003 